Research Paper

\title{
Development and validation of a comprehensive radiomics nomogram for prognostic prediction of primary hepatic sarcomatoid carcinoma after surgical resection
}

\author{
Youyin Tang ${ }^{1 *}$, Tao Zhang ${ }^{*}$, Yunuo Zhao ${ }^{3}$, Zheyu Chen ${ }^{1 凶}$ and Xuelei Ma ${ }^{3 凶}$ \\ 1. Department of Liver Surgery, Liver Transplantation Center, West China Hospital of Sichuan University, No. 37 GuoXue Alley, Chengdu 610041, China. \\ 2. West China School of Medicine, West China Hospital, Sichuan University, No. 37 GuoXue Alley, Chengdu 610041, China. \\ 3. Department of Biotherapy, West China Hospital and State Key Laboratory of Biotherapy, Sichuan University, No. 37 GuoXue Alley, Chengdu 610041, \\ China. \\ *These authors contribute equally to this work. \\ $\triangle$ Corresponding authors: Zheyu Chen, E-mail: chenzheyu@scu.edu.cn, Department of Liver Surgery, Liver Transplantation Center, West China Hospital of \\ Sichuan University, No. 37 GuoXue Alley, Chengdu 610041, China, +86 18982030423. Xuelei Ma, E-mail: drmaxuelei@gmail.com, Department of Biotherapy, \\ West China Hospital and State Key Laboratory of Biotherapy, Sichuan University, No. 37 GuoXue Alley, Chengdu 610041, China. \\ (C) The author(s). This is an open access article distributed under the terms of the Creative Commons Attribution License (https://creativecommons.org/licenses/by/4.0/). \\ See http://ivyspring.com/terms for full terms and conditions.
}

Received: 2020.09.23; Accepted: 2021.01.06; Published: 2021.02.06

\begin{abstract}
Objective: This study aimed to establish and validate a radiomics nomogram comprised of clinical factors and radiomics signatures to predict prognosis of primary hepatic sarcomatoid carcinoma (PHSC) patients after surgical resection.

Methods: In this retrospective study, 79 patients with pathological confirmation of PHSC and underwent surgical resection were recruited. A radiomics nomogram was developed by radiomics signatures and independent clinical risk factors selecting from multivariate Cox regression. All patients were stratified as high risk and low risk by nomogram. Model performance and clinical usefulness were assessed by C-index, calibration curve, decision curve analysis (DCA) and survival curve.

Results: A total of 79 PHSC were included with 1-year and 3-year overall survival rates of $63.3 \%$ and $35.4 \%$, respectively. The least absolute shrinkage and selection operator (LASSO) method selected 3 features. Multivariate Cox analysis found six independent prognostic factors. The radiomics nomogram showed a significant prediction value with overall survival (HR: $7.111,95 \% \mathrm{Cl}$ : $3.933-12.858, \mathrm{P}<0.001$ ). C-index of nomogram was 0.855 and 0.829 in training and validation set, respectively. Decision curve analysis validated the clinical utility of this nomogram. There was a significant difference in the 1-year and 3 -year survival rates of stratified high-risk and low-risk patients in the whole cohort $(30.6 \%$ vs. $90.1 \%$ and $5.6 \%$ vs. $62.4 \%$, respectively, $P<0.001$ ).

Conclusion: This radiomics nomogram serve as a potential tool for predicting prognosis of PHSC after surgical resection, and help to identify high risk patients who may obtain feeble survival benefit from surgical resection.
\end{abstract}

Key words: primary hepatic sarcomatoid carcinoma; prognosis; nomogram; radiomics; clinical decision-making

\section{Introduction}

Primary hepatic sarcomatoid carcinoma (PHSC) is a rare and complicated type of liver malignant tumor, and the reported incidence was $0.18 \%-0.7 \%$ in all liver malignancy $[1,2]$. Previous studies found that PHSC is a hybrid-type neoplasm comprised of carcinoma and sarcomatoid components, and can be mainly divided into sarcomatoid hepatocellular carcinoma (SHC), intrahepatic sarcomatoid cholangiocarcinoma (ISCC) and undifferentiated carcinoma by different carcinoma components according to World Health Organization (WHO) classification of digestive tumours [3-5]. In addition, the sarcomatoid components of PHSC were different from the conventional sarcomatoid carcinoma, such 
as osteosarcoma, hepatic follicular dendritic cell sarcoma and other sarcoma, and were usually defined as the composing of spindle cells and ultrastructurally, immunohistochemically and morphologically recognizable epithelial components $[6,7]$. In the literature review, only few series or case reports of PHSC were reported due to its rarity, and a majority of them studied the special histological or image characteristics of PHSC [5, 8-13], whereas the others discussed the clinical factors relating to its poor prognosis, with the estimating 1-year, 3-year and 5 -year overall survival rate of $40 \%-50 \%, 17 \%-21.4 \%$ and $14.3 \%-17 \%$ after surgery, respectively $[1,2]$. The prognosis of PHSC was extremely poor regarding to its histological nature of sarcomatoid carcinoma [8], however, as for the risk factors of survival, these previous prognostic studies were inconclusive because they only pay attention to the clinical factors but ignored the impact of cellular heterogeneity of PHSC on prognosis.

Radiomics is defined as quantitative depicting, which can be interpreted as extraction, interpretation and analyzing of quantitative features of digital medical image to predict clinical outcomes [14]. In a sense, radiomics can analyze the heterogeneity of tumor from the image itself [15-17]. In recent years, this non-invasive and quantitative evaluation method of tumor heterogeneity has also been developing rapidly, and has been applied in various imaging technologies [18-20]. Radiomics can be considered as a prognostic tool with outstanding potential. And Nomogram used clinical indicators or biological attributes through multiple factors regression analysis as well as used number marked line segments to predict the probability of certain events or clinical outcomes based on the values of multiple factors [21]. It converted the obscure regression equation into an intuitive and simple graph, which made the results of the prediction model easier to understand [22]. Previous study found that a radiomics nomogram comprising of clinical factors and radiomics signature can provide higher prediction value than either clinical model or radiomics model in hepatocellular carcinoma and intrahepatic cholangiocarcinoma [23-25]. However, to our knowledge, there was no radiomics nomogram regarding to the survival prediction of PHSC after surgical resection.

The goal of this study was to develop a nomogram based on enhanced computed tomographic (CT) radiomics and clinical factors to predict prognosis of patients with PHSC after surgical resection, identifying high risk patients who may obtain feeble survival benefit from surgical resection.

\section{Materials and Methods}

\section{Study design and patient selection}

This study was approved by the Ethics Committee of West China Hospital, Sichuan University. No inform consent required because of no individual information was disclosed. In this study, we retrospectively reviewed all patients with pathological proven of primary hepatic sarcomatoid carcinoma (PHSC) according to WHO classification [3, 4] and received curative-intent surgery without preoperative radiochemotherapy during January 2010 to December 2017 in West China Hospital of Sichuan University. The pathological slices of PHSC could found sarcomatoid components and liver carcinoma components. The sarcomatoid components of PHSC were composing of spindle cells and ultrastructurally, immunohistochemically and morphologically recognizable epithelial components. And other biomarkers, such as cytokeratin 7, cytokeratin 19, cytokeratin 20, epithelial membrane antigen, vimentin, HepPar1 and smooth muscle actin might be positive.

The inclusion criteria were: i) Patients had definite pathological diagnosis of PHSC and received curative-intent surgery; ii) patients had complete medical records and follow-up; iii) patient had available abdominal enhanced CT scan data before curative surgery. The exclusion criteria were: i) patients had definite pathological diagnosis of hepatic carcinosarcoma (CS), hepatocellular carcinoma (HCC) or intrahepatic cholangiocarcinoma (ICC) with sarcomatoid change or extrahepatic origin of sarcomatoid carcinoma with liver metastasis; ii) patients received any kind of radio chemotherapy (including transarterial chemoembolization) prior to CT scan or only received tumor biopsy during the surgery; iii) patients were diagnosed with other organ's malignancy (co-existing malignancy). The overall survival (OS) time was calculated between the surgery date and the date of death or last follow-up. The workflow of this study was shown in Figure 1 and the patient selection flow diagram was shown in Figure 2.

\section{CT image acquisition}

All selected patients underwent enhanced abdominal CT examinations. All the instruments for CT examination were 64-detector row scanner (Brilliance 64, Philips Medical Systems, Eindhoven, the Netherlands). CT scanning was performed with 30 to 35 seconds for arterial-phase and 60 to 70 seconds for portal venous phase. Other specific CT scan parameters were reported in previous studies [26]. 
A Patient selection and radiomics feature extration
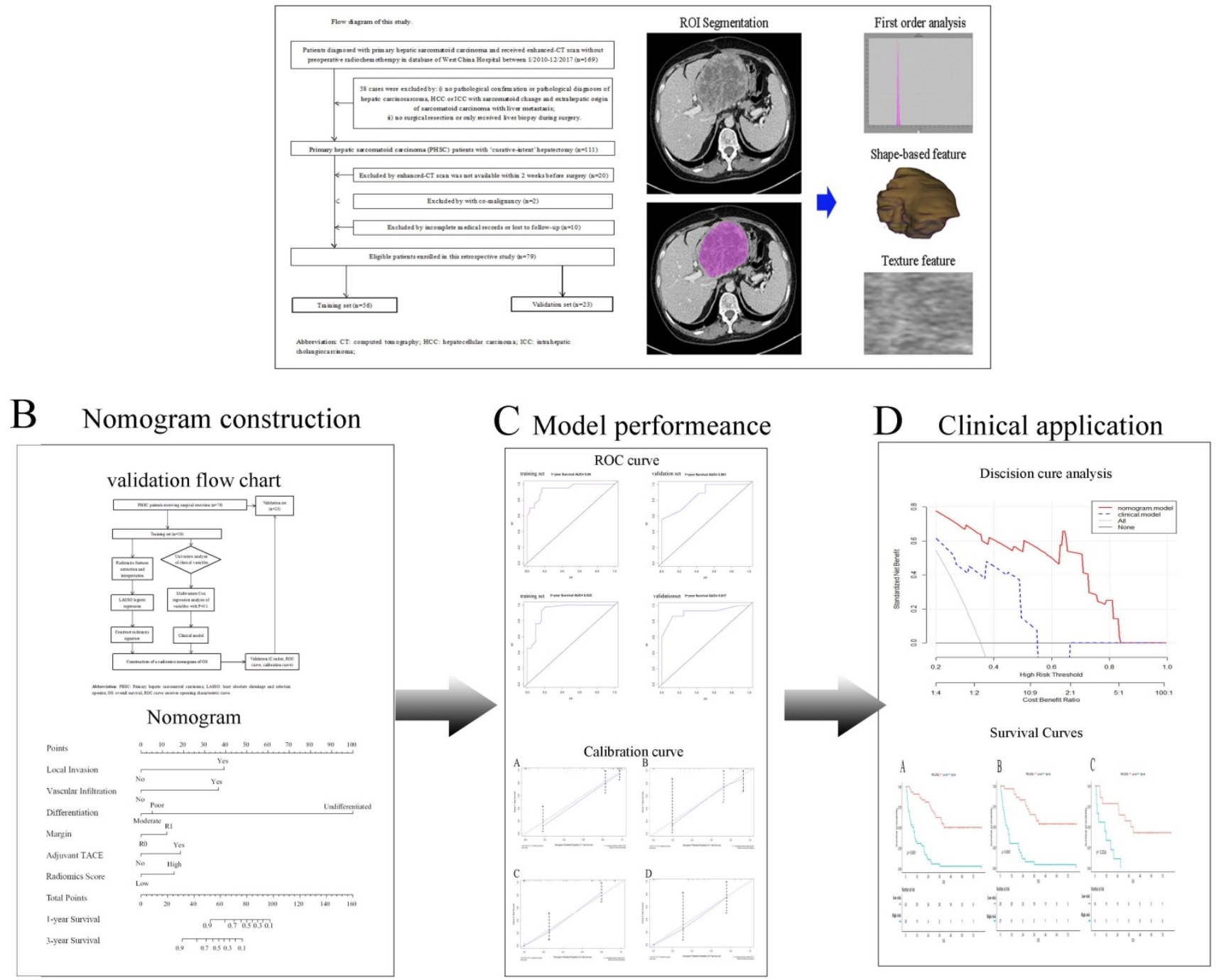

Figure 1. Study workflow. A Patients selection and Radiomics features extraction; B Construction of nomogram; C Comparison of model performance; D Clinical decision analysis and survival comparison. CT: computed tomographic; HCC: hepatocellular carcinoma; ICC: introhepatic cholangiocarcinoma; ROI: region of interest; TACE: transcatheter arterial chemoembolization.

\section{Radiomics features extraction and radiomics score model building}

As primary hepatic sarcomatoid carcinoma was mainly presented as a hypovascular liver mass with low intensity and more clearly defined in the venous phase. The venous phase CT imaging data was retrieved and loaded into the Local Image Features Extraction (LIFEx) software (v3.74, CEA-SHFJ, Orsay, France) to quantify pathological lesion segmentation and automated quality features [27]. The ROIs were depicted freehand within the tumor lesion in venous phase images of enhanced CT by two independent radiologists. For large mass and multiple mass, ROIs were depicted within all the tumor lesions.

All enrolled patients were randomly divided into training set and validation set, with a ratio of 7:3. The Least Absolute Shrinkage and Selection Operator
(LASSO) Cox regression method was used for selecting the appropriate radiomics features (features with non-zero coefficients) in the training set. The radiomics scoring model was obtained by linear combination of selected features based on non-zero coefficients of themselves. By means of the largest Youden index [28], we transformed the calculated radiomics scores from continuous variables into a categorical variable, and divide them into two categories: high score and low score.

\section{Selection of clinical factors and establishment of clinical model}

Patients' baseline characteristics, surgical information as well as pathological information were respectfully reviewed from the hospital record system. Univariate Cox analysis and step-wise multivariate Cox regression analysis were all used to 
identify independent prognostic risk factors from these clinical variables. The clinical model was established by selected independent prognostic risk factors with their coefficients.

\section{Development and validation of nomogram}

The construction of nomogram was based on the established radiomics scoring model and the independent clinical risk factors obtained from multivariate Cox analysis. The performance of nomogram was validated by calibration curve, concordance index (C-index), receiver operating characteristic (ROC) curve, decision curve analysis (DCA) $[29,30]$.

\section{Statistical analysis}

All clinical variables were displayed as mean \pm standard deviation (continuous variable) and frequency with percentage (categorical variable). We used the Chi-square test and Student's $t$ test to find out the difference between training and validation set. The survival curve was described by Kaplan-Meier method. In univariate Cox analysis, variables with $p$ value $<0.1$ were further selected for multivariate Cox regression analysis, and $p<0.05$ was considered statistically significant [31]. An estimated 15 patients (the real cohort of high risk and low risk patients were 36 and 43 , respectively) would be needed to provide $90 \%$ power for 3-year overall survival log-rank test with a two-sided a of 0.05 (Supplement Table 2).All statistical analyses were conducted by SPSS, version 20.0 (IBM Corporation, Armonk, NY, USA) and R statistical software, version 4.0.0 (The $\mathrm{R}$ Foundation). The packages used in $\mathrm{R}$ software are glmnet, cmprsk, rms, survival, rmda and devtools.

\section{Results}

\section{Patient characteristics}

Finally, a total of 79 eligible patients with pathological confirmation of PHSC were included in the study. The number of men and women was 43 and 36 , respectively. The mean age of all patients was 51.1 years old. The median follow-up time was $30.1 \pm 4.6$ (95\%CI: 21.1-38.9) months. The 1-year and 3-year overall survival rates were $63.3 \%$ and $35.4 \%$, respectively. The median survival time was 23.4 months of the whole set. The 1-year and 3-year overall survival rates in training set were $64.3 \%$ and $35.7 \%$, respectively, contrast to those of $60.9 \%$ and $34.8 \%$ in validation set, respectively. The median survival time in training and validation set were 22.9 months and 24.5 months, respectively. And no significant difference of baseline characteristics was observed between training and validation set. All patients' baseline characteristics were summarized in Table 1.

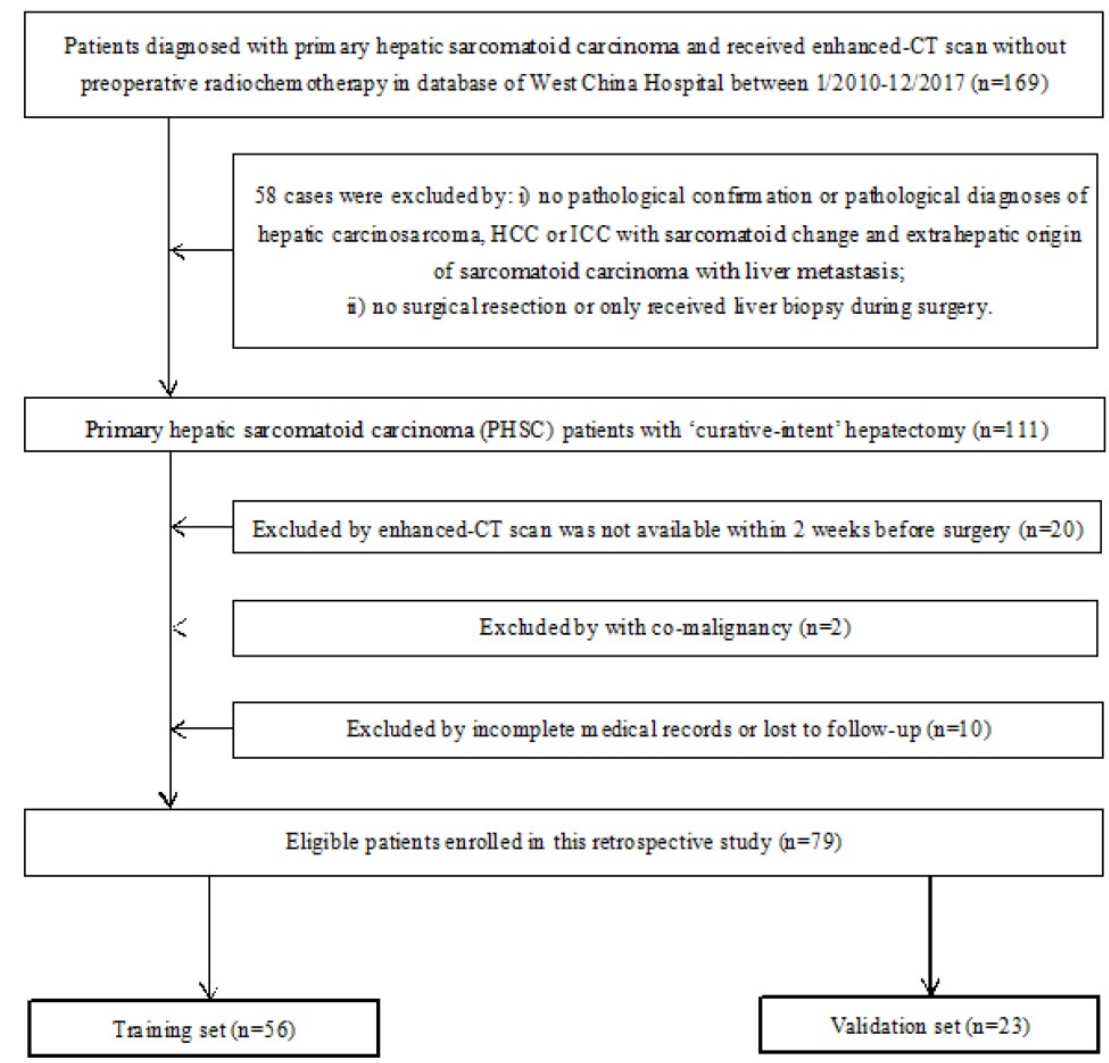

Figure 2. Flow diagram of patient selection of this study. CT: computed tomographic; HCC: hepatocellular carcinoma; ICC: introhepatic cholangiocarcinoma. 
Table 1. Characteristics of patients with PHSC after surgical resection

\begin{tabular}{|c|c|c|c|c|c|c|c|c|}
\hline \multirow[t]{2}{*}{ Characteristic } & \multicolumn{3}{|l|}{ PHSC patients } & \multirow[t]{2}{*}{$P$} & \multirow{2}{*}{\multicolumn{4}{|c|}{$\begin{array}{l}\text { univariate Cox analysis and multivariate Cox } \\
\text { analysis. Univariate analysis revealed } 9 \text { potential }\end{array}$}} \\
\hline & $\begin{array}{l}\text { Entire set } \\
(\mathrm{n}=79)\end{array}$ & $\begin{array}{l}\text { Training set } \\
(n=56)\end{array}$ & $\begin{array}{l}\text { Validation set } \\
(\mathrm{n}=23)\end{array}$ & & & & & \\
\hline Sex, n (\%), male & $43(54.4)$ & $28(50)$ & $15(65.2)$ & 0.22 & \multirow{2}{*}{\multicolumn{4}{|c|}{ predictors: CA19-9 level, AJCC TNM staging, }} \\
\hline Age $(y)$, mean $\pm S D$ & $51.1 \pm 13.4$ & $50.8 \pm 13.5$ & $51.8 \pm 13.4$ & 0.765 & & & & \\
\hline $\mathrm{ALT}(\mathrm{U} / \mathrm{L})$, mean $\pm \mathrm{SD}$ & $54.5 \pm 44.6$ & $55.2 \pm 49.2$ & $49.3 \pm 31.3$ & 0.53 & \multicolumn{4}{|c|}{ Child-Pugh classification, number of tumors, } \\
\hline $\mathrm{AST}(\mathrm{U} / \mathrm{L})$, mean $\pm \mathrm{SD}$ & $52.0 \pm 35.8$ & $50.6 \pm 36.7$ & $53.4 \pm 33.8$ & 0.58 & \multirow{2}{*}{\multicolumn{4}{|c|}{$\begin{array}{l}\text { differentiation, local invasion, vascular infiltration, } \\
\text { surgical margin and adjuvant transcatheter arterial }\end{array}$}} \\
\hline $\begin{array}{l}\text { Serum albumin }(\mathrm{g} / \mathrm{L}) \\
\text { mean } \pm \mathrm{SD}\end{array}$ & $41.3 \pm 4.8$ & $41.5 \pm 4.9$ & $40.9 \pm 4.4$ & 0.61 & & & & \\
\hline $\begin{array}{l}\text { TBIL (umol/L), mean } \pm \\
\text { SD }\end{array}$ & $15.2 \pm 6.3$ & $15.7 \pm 6.7$ & $14.1 \pm 5.2$ & 0.26 & \multirow{2}{*}{\multicolumn{4}{|c|}{$\begin{array}{l}\text { chemoembolization (TACE). The } \mathrm{p} \text {-values of these } \\
\text { variables were all less than } 0.1 \text {. We put these nine } \\
\text { variables into the multivariate Cox regression model, }\end{array}$}} \\
\hline $\begin{array}{l}\text { Prothrombin time }(\mathrm{s}) \text {, } \\
\text { mean } \pm \text { SD }\end{array}$ & $12.5 \pm 1.9$ & $12.4 \pm 1.9$ & $12.7 \pm 1.7$ & 0.48 & & & & \\
\hline $\begin{array}{l}\operatorname{AFP}(\mathrm{ng} / \mathrm{ml}), \text { mean } \pm \\
\mathrm{SD}\end{array}$ & $194.7 \pm 333.7$ & $239.6 \pm 378.1$ & $85.3 \pm 141.2$ & 0.06 & \multicolumn{4}{|c|}{$\begin{array}{l}\text { and finally got five variables with } \mathrm{p} \text {-value }<0.05 \text {. } \\
\text { These variables were considered as independent risk }\end{array}$} \\
\hline $\begin{array}{l}\text { CA19-9 }(\mathrm{U} / \mathrm{ml}) \text {, mean } \pm \\
\text { SD }\end{array}$ & $45.4 \pm 127.8$ & $53.0 \pm 150.4$ & $27.0 \pm 28.9$ & 0.22 & \multirow{2}{*}{\multicolumn{4}{|c|}{$\begin{array}{l}\text { prognostic factors regarding to OS for patients with } \\
\text { PHSC after surgical resection. Table } 2 \text { showed the }\end{array}$}} \\
\hline $\begin{array}{l}\mathrm{CEA}(\mathrm{ng} / \mathrm{ml}), \text { mean } \pm \\
\mathrm{SD}\end{array}$ & $3.1 \pm 3.9$ & $3.4 \pm 4.5$ & $2.5 \pm 1.5$ & 0.17 & & & & \\
\hline $\begin{array}{l}\text { Child-Pugh class, n, } \\
\text { A/B }\end{array}$ & $72 / 7$ & $51 / 5$ & $21 / 2$ & 0.97 & \multirow{4}{*}{\multicolumn{4}{|c|}{$\begin{array}{l}\text { details of univariate and multivariate analysis results. } \\
\text { Table 2. Univariate analysis and multivariate Cox regression } \\
\text { analysis of clinical factors in the training cohort }\end{array}$}} \\
\hline $\begin{array}{l}\text { Tumor size }(\mathrm{cm}) \text {, mean } \\
\pm \mathrm{SD}\end{array}$ & $7.1 \pm 4.0$ & $6.6 \pm 3.9$ & $7.1 \pm 3.2$ & 0.52 & & & & \\
\hline Number of tumor, $\mathrm{n}(\%)$ & & & & 0.79 & & & & \\
\hline Single & $46(58.2)$ & $33(58.9)$ & $13(56.5)$ & & & & & \\
\hline Multiple & $33(41.8)$ & $23(41.1)$ & $10(43.5)$ & & \multirow{2}{*}{ Variable } & \multirow{2}{*}{$\begin{array}{l}\text { Univariate analysis } \\
(p \text { value })\end{array}$} & \multirow{2}{*}{$\begin{array}{l}\text { Multivariate } \\
\text { analysis HR }(95 \% \mathrm{CI})\end{array}$} & \multirow{2}{*}{$\begin{array}{l}p \\
\text { value }\end{array}$} \\
\hline $\begin{array}{l}\text { Capsule formation, } \mathrm{n} \\
(\%)\end{array}$ & $8(10.1)$ & $7(12.5)$ & $1(4.3)$ & 0.28 & & & & \\
\hline Local invasion, $\mathrm{n}(\%)$ & $28(35.4)$ & $19(33.9)$ & $9(39.1)$ & 0.67 & Sex & 0.781 & & \\
\hline Vascular invasion, $\mathrm{n}$ & $17(21.5)$ & $13(23.2)$ & $4(17.3)$ & 0.56 & Age, years & 0.106 & & \\
\hline$(\%)$ & & & & & Hepatitis & 0.889 & & \\
\hline Bile duct invasion, $\mathrm{n}$ & $10(12.7)$ & $7(12.5)$ & $3(13.0)$ & 0.95 & ALT level, U/L & 0.896 & & \\
\hline 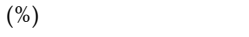 & & & & & AST level, U/L & 0.787 & & \\
\hline Lymph node & $9(11.4)$ & 7 (12.5) & $2(8.6)$ & 0.61 & ALB level, g/L & 0.225 & & \\
\hline metastasis, n (\%) & & & & & TBIL level, umol/L & 0.806 & & \\
\hline $\begin{array}{l}\text { Cancerous component, } \\
\text { HCC/ICC }\end{array}$ & $43 / 36$ & $31 / 2 b$ & $12 / 11$ & 0.80 & AFP level, ng/m & 0.779 & & \\
\hline Differentiation, n (\%) & & & & 0.59 & $\mathrm{PT}, \mathrm{s}$ & 0.685 & & \\
\hline Moderately & $8(10.1)$ & $5(8.9)$ & $3(13.0)$ & & CA19-9 level, U/ml & 0.059 & $1.001(1.000-1.003)$ & 0.067 \\
\hline Poorly & $35(44.3)$ & $27(48.2)$ & $8(34.8)$ & & CEA level, ng/ml & 0.220 & & \\
\hline Undifferentiated & $4(5.1)$ & $3(5.4)$ & $1(4.3)$ & & Tumor size, cm & 0.353 & & \\
\hline NA & $32(40.5)$ & $21(37.5)$ & $11(47.8)$ & & 8th AJCC staging & 0.001 & & \\
\hline 8th AJCC staging, n (\%) & & & & 0.72 & $\mathrm{I}+\mathrm{II}$ & & Ref & \\
\hline $\mathrm{I}+\mathrm{II}$ & $40(50.6)$ & $28(50.0)$ & $12(52.2)$ & & III & & Ref & \\
\hline IIIA+IIIB & $30(38.0)$ & $21(37.5)$ & $9(39.1)$ & & IVA & & $1.651(0.633-4.309)$ & 0.305 \\
\hline IVA & $9(11.4)$ & $7(12.5)$ & $2(8.7)$ & & Child-Pugh classification & 0.011 & & \\
\hline Extent of hepatectomy, & & & & 0.17 & A & & Ref & \\
\hline $\mathrm{n}(\%)$ & & & $10(435)$ & & B & & $1.221(0.480-3.106)$ & 0.676 \\
\hline $\begin{array}{l}\text { Minor liver resection } \\
\text { Major liver resection }\end{array}$ & $\begin{array}{l}45(5 / .0) \\
31(39.2)\end{array}$ & $\begin{array}{l}35(62.5) \\
19(33.9)\end{array}$ & $\begin{array}{l}10(43.5) \\
12(52.2)\end{array}$ & & Number of tumors & 0.001 & & \\
\hline Liver transplantation & $3(3.8)$ & $2(3.6)$ & $1(4.3)$ & & Single & & Ref & \\
\hline Anatomy resection, $\mathrm{n}$ & $16(20.3)$ & $11(19.6)$ & $5(21.7)$ & 0.84 & Multiple (>1) & & $0.769(0.370-1.599)$ & 0.270 \\
\hline$(\%)$, yes & & & & & Differentiation & 0.003 & & \\
\hline Transfusion, n (\%), yes & $14(17.7)$ & $10(17.8)$ & $4(17.4)$ & 0.96 & moderate & & Ref & \\
\hline Blood loss (ml), mean \pm & $660.8 \pm 778.3$ & $626.8 \pm 651.4$ & $743.5 \pm 1037.7$ & 0.62 & Poor & & Ref & \\
\hline SD & & & & & Undifferentiated & & 1.597 (1.143-2.237) & 0.006 \\
\hline Margin, n (\%) & & & & 0.95 & Capsule formation & 0.232 & & \\
\hline R0 & $69(87.3)$ & $49(87.5)$ & $20(87.0)$ & & Local invasion & 0.001 & & \\
\hline R1 & $10(12.7)$ & $7(12.9)$ & $3(13.0)$ & & No & & Ref & \\
\hline Adjuvant TACE, n (\%) & & & & 0.98 & Yes & & 3.159 (1.675-5.957) & $<0.001$ \\
\hline Yes & $17(21.5)$ & $12(21.4)$ & $5(21.7)$ & & Vascular infiltration & 0.047 & & \\
\hline No & $62(78.5)$ & 44 (78.3) & $18(78.3)$ & & No & & Ref & \\
\hline $\begin{array}{l}\text { Hospital stay }(\mathrm{d}), \text { mean } \\
\pm \mathrm{SD}\end{array}$ & $13.5 \pm 7.6$ & $13.2 \pm 7.6$ & $14.3 \pm 7.5$ & 0.59 & Yes & & $4.417(2.112-9.236)$ & $<0.001$ \\
\hline Total hospitalization & $7913.0 \pm 8367.5$ & $7850.8 \pm 8185.8$ & $8064.5 \pm 8982.2$ & 0.92 & Bile duct invasion & 0.816 & & \\
\hline expenses (USD), mean \pm & & & & & Lymph node metastasis & 0.012 & & \\
\hline SD & & & & & Cancerous & 0.503 & & \\
\hline Abbreviation: PHSC: pri & imary hepatic s & omatoid carci & a; SD: standar & & component,HCC/ICC & & & \\
\hline & aminotransfera & AS1: aspartat & inotransferase & BIL: & Anatomy resection & 0.401 & & \\
\hline total bilirubin; AFP: alph & ha-fetoprotein; & A: carcinoeml & ic antigen; $\mathrm{HC}$ & & Extent of hepatectomy & 0.281 & & \\
\hline $\begin{array}{l}\text { hepatocellular carcinom } \\
\text { applicable; AJCC: Ameri } \\
\text { arterial chemoembolizat }\end{array}$ & $\begin{array}{l}\text { ican joint Comn } \\
\text { tion: USD: Unite }\end{array}$ & ttee on Cancer; & ACE: transcathe & & $\begin{array}{l}\text { Intraoperative blood } \\
\text { transfusion }\end{array}$ & 0.366 & & \\
\hline
\end{tabular}

Clinical independent preoperative prognostic factors

We included a total of 28 clinical variables in Cox analysis. Univariate analysis revealed 9 potential predictors: CA19-9 level, AJCC TNM staging, differentiation, local invasion, vascular infiltration, surgical margin and adjuvant transcatheter arterial chemoembolization (TACE). The p-values of these variables into the multivariate Cox regression model, These variables were considered as independent risk prognostic factors regarding to OS for patients with PHSC after surgical resection. Table 2 showed the details of univariate and multivariate analysis results.

Table 2. Univariate analysis and multivariate Cox regression analysis of clinical factors in the training cohor 


\begin{tabular}{llll}
\hline Variable & $\begin{array}{l}\text { Univariate analysis } \\
(p \text { value })\end{array}$ & $\begin{array}{l}\text { Multivariate } \\
\text { analysis HR }(95 \% \mathrm{CI})\end{array}$ & $\begin{array}{l}p \\
\text { value }\end{array}$ \\
\hline $\begin{array}{l}\text { Blood loss, ml } \\
\text { Surgical margin }\end{array}$ & 0.674 & & \\
R0 & 0.034 & Ref & \\
R1 & & $3.232(1.486-7.027)$ & 0.003 \\
Adjuvant TACE & 0.001 & & \\
No & & Ref & \\
Yes & & $2.327(1.115-4.858)$ & 0.025 \\
\hline
\end{tabular}

Abbreviation: ALT: alanine aminotransferase; AST: aspartate aminotransferase; TBIL: total bilirubin; AFP: alpha-fetoprotein; PT: prothrombin time; CEA: carcinoembryonic antigen; NA: not applicable; AJCC: American joint Committee on Cancer; HCC: hepatocellular carcinoma; ICC: introhepatic cholangiocarcinoma; TACE: transcatheter arterial chemoembolization; ref: reference.

\section{Feature selection and construction of radiomics score}

Among the 49 radiomics features, 3 non-zero coefficient features related to prognosis were selected by LASSO regression model from training set. We combined these features linearly according to their non-zero coefficients, and finally calculated the radiomics scores of each patient. The formulas of these 3 features and their non-zero coefficients were shown in Supplement Table 1.

\section{Construction and validation of the nomogram}

We built a comprehensive radiomics model with five previously estimated clinical variables and radiomics score, and integrated them into a nomogram. This nomogram was constructed to predict each patient's probability of survival at 1 and 3 years after surgical resection (Figure 3 ). We applied C-index and calibration curve (Figure 4) to determine the predictive accuracy and discriminative ability of the nomogram. The C-index was $0.855(95 \% \mathrm{CI}$, $0.798-0.912)$ in training set and 0.829 (95\% CI, $0.702-0.956)$ in validation set. For 1 -year OS, the area under curve (AUC) was 0.940 in training set and 0.861 in validation set. For 3-year OS, AUC of training set and validation set were 0.932 and 0.917 , respectively.

\section{Prognosis comparison and risk stratification}

We added the scores of each nomogram variable to get the total risk score of each patient. All patients were then divided into high-risk group and low-risk group according to the cut-off value. And there were 36 high risk patients and 43 low risk patients. The result of Kaplan-Meier curve showed significantly survival difference between high-risk and low-risk patients stratified by the radiomics nomogram in entire cohort (1-year and 3-year survival rate, high risk versus low risk, $30.6 \%$ versus $90.1 \%$ and $5.6 \%$ versus $62.4 \%$, respectively, $\mathrm{P}<0.001)$. The same results were also found in training set and validation set (Figure 5). The hazard ratios of nomogram, radiomics score and clinical models are shown in Table 3 . This nomogram obtained the highest HR value than clinical model and radiomics score. An example of using radiomics nomogram to predict survival probability of a 35 years old male patient after surgical resection was showed in Supplement Figure 1.

\section{Decision curve analysis in radiomics nomogram and clinical model}

The nomogram showed a better performance than clinical model for almost threshold probabilities. Especially when the threshold value is less than 0.8, the net benefit of nomogram was significantly higher than that of clinical model. When the threshold value increased, the net benefits of two models were closer. Decision curve analysis results for the nomogram and clinical model were presented in Figure 6.

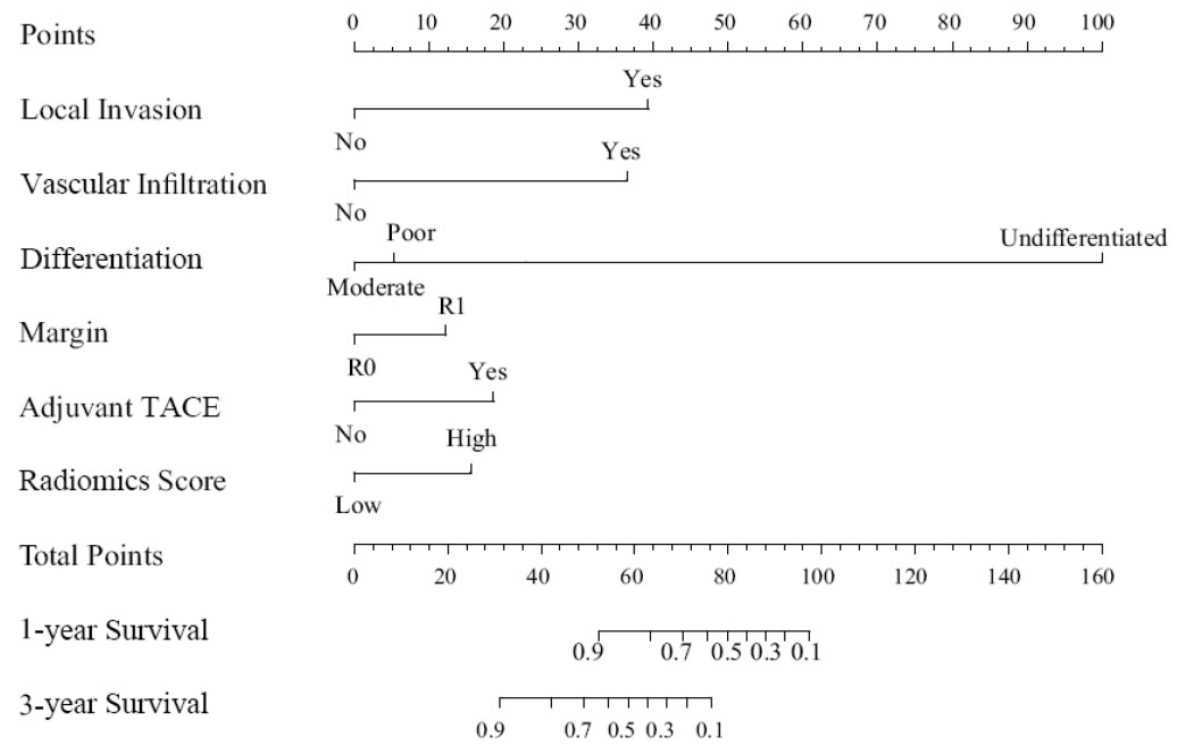

Figure 3. Nomogram for 1 and 3 year OS in patients with PHSC after surgical resection. OS: overall survival; PHSC: primary hepatic sarcomatoid carcinoma; TACE: transcatheter arterial chemoembolization. 

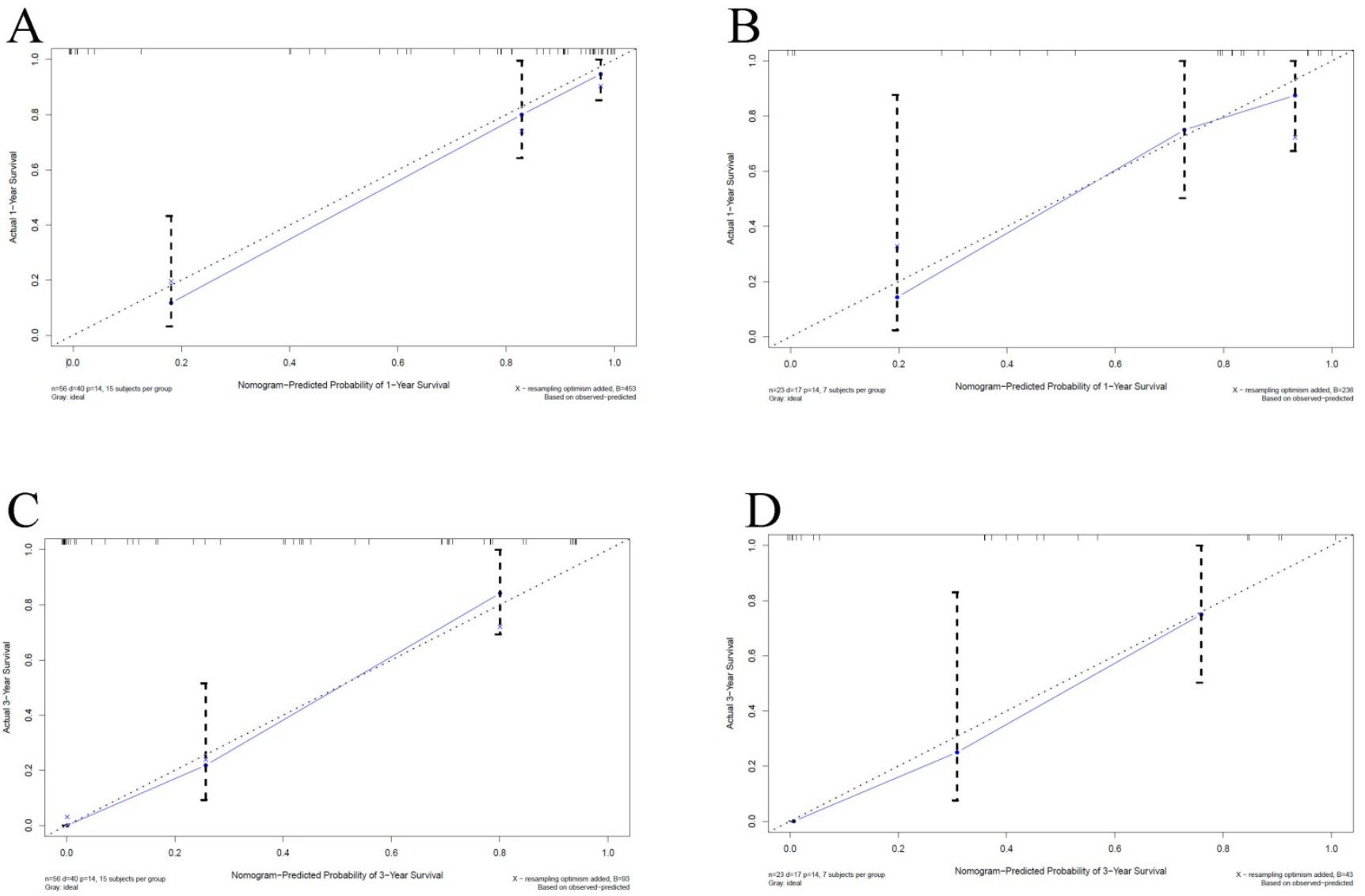

Figure 4. Calibration curves for overall survival (OS) at 1-year and 3-year in patients with PHSC after surgical resection. A: 1-yearsurvival rate in training set; B: 1-yearsurvival rate in validation set; C: 3-yearsurvival rate in training set; D: 3-yearsurvival rate in validation set. The horizontal axis was the survival rate predicted by the nomogram, and the vertical axis was the actual survival rate. The dashed line indicates the predicting survival rate completely fits the actual survival rate. In the training set and validation set, the prediction results of the nomogram were close to the actual results of 1-year and 3-year OS, showed the calibration curve was in good agreement. PHSC: primary hepatic sarcomatoid carcinoma.

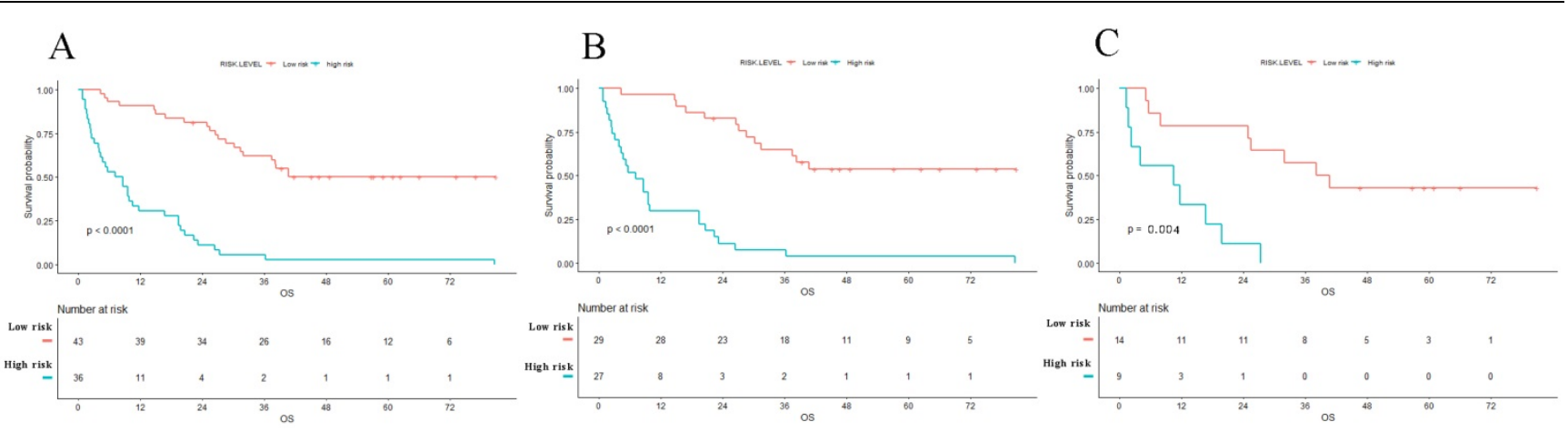

Figure 5. Kaplan-Meier survival curves of OS in patients with PHSC after surgical resection in entire (A) set, training set (B) and validation set (C). Patients with low risk were associated with better survival $(\mathrm{p}<0.001)$. OS: overall survival; PHSC: primary hepatic sarcomatoid carcinoma.

Table 3. Comparison of hazard ratio of three models

\begin{tabular}{lll}
\hline Model & HR $(95 \% \mathrm{CI})$ & $p$-value \\
\hline $\begin{array}{l}\text { Radiomics score } \\
\text { Low risk }\end{array}$ & Ref & 0.009 \\
$\begin{array}{l}\text { High risk } \\
\text { Clinical model }\end{array}$ & $2.047(1.198-3.498)$ & \\
Low risk & Ref & $<0.001$ \\
High risk & $5.205(2.644-10.648)$ & \\
Nomogram & & $<0.001$ \\
Low risk & Ref & \\
High risk & $7.111(3.933-12.858)$ & \\
\hline
\end{tabular}

Abbreviation: HR: hazard ratio, Ref: reference.

\section{Discussion}

PHSC is a sophisticated type of liver malignancy with poor prognosis $[1,2,8,32,33]$. Due to its poor prognosis, it is very important to predict the overall survival for PHSC patients. In this study, we found that positive surgical margin, vascular infiltration, local invasion, differentiation, adjuvant TACE and image score were all independent factors relating to prognosis through univariate and multivariate analysis. We developed a comprehensive 
nomogram based on these independent risk factors, and then stratified patients into high risk and low risk set. There was significantly different survival time between high risk and low risk patients (average survival time, high risk versus low risk, 12.1 months versus 52.7 months, $\mathrm{P}<0.001)$. To our knowledge, this was the first comprehensive CT-based radiomics nomogram for predicting overall survival of PHSC after curative-intent surgery.

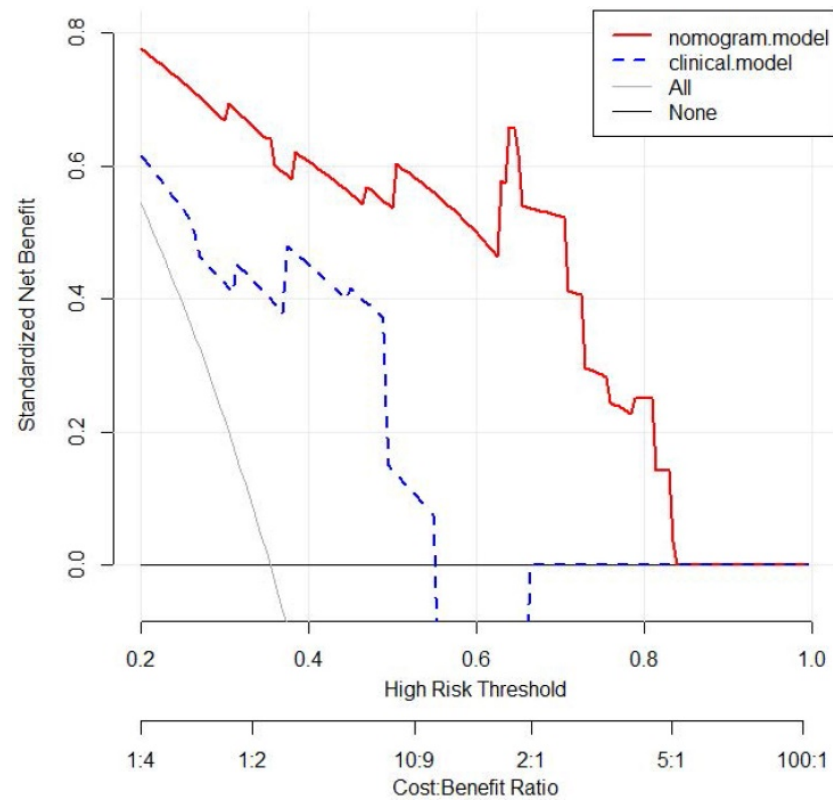

Figure 6. Decision curve analysis for 3-year OS of nomogram and clinical model in the validation set. The $y$-axis measures the net benefit. The red line represents the nomogram. The blue dotted line represents the clinical model. The gray dotted line represents the assumption that all patients dead. The black dotted line represents the assumption that no patients dead. OS: overall survival.

Curative surgery can obviously prolong the overall survival of PHSC, and radical resection can obtain significant higher survival time than palliative resection. The median survival time was reported to 15.6-20.5 months for patients who received radical resection contrast to 7.6-7.9 months for those underwent palliative resection [1, 2]. In the present study, the median survival time for $\mathrm{R} 0$ resection was 25.5 months contrast to 10.2 months of R1 resection $(p=0.017)$. In further, previous studies reported vascular infiltration and local invasion were occurred more often in PHSC than conventional hepatocellular carcinoma and were associated with worse prognosis $[1,2,13,34,35]$. In the present study, vascular infiltration and local invasion were found in $21.5 \%$ and $35.4 \%$ of all patients, respectively, which were similar to the reported incidence of $28.6 \%$ and $21.4 \%$, respectively [2]. Besides, high incidence of vascular infiltration and local invasion could result in significantly worse prognosis, with reported median disease-free survival time of 3.2 months and 3.6 months in patients with vascular infiltration and local invasion, respectively. In this study, we found that patients with vascular infiltration suffered significantly lower survival time (median survival time, vascular infiltration versus no vascular infiltration, 11.7 months versus 25.5 months, $\mathrm{P}=0.044$ ) than those with no vascular infiltration. Besides, the median survival time of patients with local invasion were significantly lower than patients without local invasion (local invasion versus no local invasion, 32.0 months versus 12.6 months, $\mathrm{P}<0.001$ ).

Tumor differentiation was associated with prognosis of liver tumor; high grade of differentiation can lead to poor prognosis. Previous study revealed that the sarcomatoid hepatocellular carcinoma was associated with more advanced histopathological grade than conventional hepatocellular carcinoma [36]. Moreover, a population-based study also found sarcomatoid hepatocellular carcinoma were relating to worse tumor grade than conventional HCC through propensity score matching [37]. In this study, patients with advanced differentiation grade (poor differentiation and undifferentiation) were about $49.4 \%$ of all patients, which was similar to the previous reported incidence of 52.9\% [37]. In addition, we found that advanced differentiation grade was associated with worse prognosis than moderate differentiation (average survival time, advanced grade versus moderate grade, 34.0 months versus 55.6 months, $\mathrm{P}=0.019)$. The survival curves stratified by surgical margin, vascular invasion, local invasion, and differentiation grade were shown in Supplement Figure 2.

Radiomics is a renewed objective and quantitative evaluation method of cancer heterogeneity, which was better than sampling biopsy which could assess only a small part of the tumor [38, 39]. Many previous studies had confirmed the flexibility and effectiveness of $\mathrm{CT}$ radiomics in recurrence and prognostic prediction of liver malignancies [40-44]. Moreover, the nomogram based on radiomics may obtain higher prediction value than clinical model only [23-25]. In the present study, we integrated three radiomics features which were extracted from 49 radiomics features and five clinical independent risk factors into a comprehensive radiomics nomogram for prognostic prediction. The DCA showed that the net benefit was higher in radiomics nomogram than clinical model when the threshold less than 0.8. Besides, when compared the model performance with three models, we also found that the HR in nomogram was higher than that either in radiomics score or clinical model. Our results were in line with the previous studies. 
Surprisingly, the present study found that patient cannot obtain survival benefit in adjuvant TACE. Previous studies found that PHSC was mainly present as a hypovascular liver mass with low intensity [5, 8, 45, 46], albeit another study insisted that PHSC may serve as a hypervascular mass [2]. The nature of hypovascularity implied that TACE might have feeble survival benefit on these patients. In the contrary, patient who received TACE often associated with advanced tumor stage. In the present study, we found that adjuvant TACE was a hazard factor associated with worse prognosis (average survival time, TACE versus no TACE, 11.0 months versus 40.2 months, $\mathrm{P}<0.01)$. The result was agreed with the previous study of Wang et.al which reported the average survival time in TACE +operation and operation without TACE was 10.6 months and18.3 months, respectively [1]. The possible reason might be hypovascular nature of tumor and the lack of appropriate selection standard for TACE in PHSC patients. Thus, further studies focus on identifying appropriate patients for additional adjuvant TACE for PHSC after surgical resection could be conducted to verify the results.

This study still has some limitations. First, the nature of retrospective study may generate selective and withdraw bias. Second, this study was absence of external validation. Thus, future prospective and multicenter studies should be conducted to validate the results. Third, although PHSC was considered as a single tumor mixed with carcinoma and sarcomatoid components, the proportion of each component may be various in each patient. However, it was very difficult to clearly evaluate the proportion of each component in current immunohistochemistry. Thus, further radiomics studies referred to the features of high-quality histopathological images should be conducted to reduce this bias microcosmically.

\section{Conclusion}

PHSC often presented with poor prognosis. The radiomics nomogram established in this study may be useful in predicting the overall survival rate of PHSC patients after curative-intent surgery and help to identify high risk patients who may obtain feeble survival benefit from surgical resection.

\section{Key Points}

- The radiomics nomogram showed good performance for prediction of overall survival in primary hepatic sarcomatoid carcinoma after surgical resection.

- The prognosis of high-risk and low-risk patients was significantly different.
- The radiomics nomogram could benefit to make decision in identifying high risk patients.

\section{Abbreviations}

PHSC: primary hepatic sarcomatoid carcinoma; DCA: decision curve analysis; LASSO: least absolute shrinkage and selection operator; HR: hazard ratio; SD: standard deviation; ALT: alanine aminotransferase; AST: aspartate aminotransferase; TBIL: total bilirubin; AFP: alpha-fetoprotein; CEA: carcinoembryonic antigen; SHC: sarcomatoid hepatocellular carcinoma; ISCC: intrahepatic sarcomatoid cholangiocarcinoma; WHO: World Health Organization; CT: computed tomographic; HCC: hepatocellular carcinoma; ICC: introhepatic cholangiocarcinoma; CS: carcinosarcoma; ROC: receiver operating characteristic; NA: not applicable; OS: overall survival; AJCC: American joint Committee on Cancer; TACE: transcatheter arterial chemoembolization; AUC: area under curve; USD: United States dollar.

\section{Supplementary Material}

Supplementary figures and tables. http://www.medsci.org/v18p1711s1.pdf

\section{Acknowledgements}

\section{Ethics approval and informed consent}

This study was approved by the Ethics Committee of West China Hospital of Sichuan University. And inform consent was waived because no individual information was disclosed in this study.

\section{Availability of data and material}

The clinical data in this study is available from the corresponding author on reasonable request.

\section{Author contributions}

Study concept and design: Youyin Tang, Tao Zhang; Acquisition of data: Youyin Tang; Analysis and interpretation: Tao Zhang and Yunuo Zhao; Draft the manuscript and preliminary revise: Youyin Tang, Tao Zhang; Study supervision and final approval: Zheyu Chen, Xuelei Ma.

\section{Competing Interests}

The authors have declared that no competing interest exists.

\section{References}

1. Wang QB, Cui BK, Weng JM, Wu QL, Qiu JL, Lin XJ. Clinicopathological characteristics and outcome of primary sarcomatoid carcinoma and carcinosarcoma of the liver. Journal of gastrointestinal surgery : official journal of the Society for Surgery of the Alimentary Tract. 2012; 16: 1715-26.

2. Lu J, Zhang J, Xiong XZ, Li FY, Ye H, Cheng Y, et al. Primary hepatic sarcomatoid carcinoma: clinical features and prognosis of 28 resected cases. Journal of cancer research and clinical oncology. 2014; 140: 1027-35. 
3. Bosman FT, Carneiro F, Hruban RH, Theise ND. WHO classification of tumours of the digestive system: World Health Organization. 2010.

4. Nagtegaal ID, Odze RD, Klimstra D, Paradis V, Rugge M, Schirmacher P, et al. The 2019 WHO classification of tumours of the digestive system. Histopathology. 2020; 76: 182-8.

5. Seo N, Kim MJ, Rhee H. Hepatic sarcomatoid carcinoma: magnetic resonance imaging evaluation by using the liver imaging reporting and data system. European radiology. 2019; 29: 3761-71.

6. Rossi G, Cavazza A, Sturm N, Migaldi M, Facciolongo N, Longo L, et al. Pulmonary carcinomas with pleomorphic, sarcomatoid, or sarcomatous elements: a clinicopathologic and immunohistochemical study of 75 cases. The American journal of surgical pathology. 2003; 27: 311-24.

7. Rosai J. Rosai and Ackerman's Surgical Pathology Mosby. Elsevier Inc. 2004.

8. Shi D, Ma L, Zhao D, Chang J, Shao C, Qi S, et al. Imaging and clinical features of primary hepatic sarcomatous carcinoma. Cancer imaging : the official publication of the International Cancer Imaging Society. 2018; 18: 36.

9. Leng Q, Xiang XI, Tang Y, Yang Y, Qiu LI. Primary hepatic sarcomatoid carcinoma: A case report. Experimental and therapeutic medicine. 2015; 10: $1145-8$.

10. Bilgin M, Toprak H, Bilgin SS, Kondakci M, Balci C. CT and MRI findings of sarcomatoid cholangiocarcinoma. Cancer imaging : the official publication of the International Cancer Imaging Society. 2012; 12: 447-51.

11. $\mathrm{M} \mathrm{K}, \mathrm{Y} \mathrm{K}, \mathrm{N} \mathrm{Y}, \mathrm{H} \mathrm{Y}, \mathrm{S} \mathrm{T}, \mathrm{AH} \mathrm{K}$, et al. Intrahepatic sarcomatoid cholangiocarcinoma. Journal of gastroenterology. 2003; 38: 1097-101.

12. Sintra S, Costa R, Filipe C, Simão A. Intrahepatic sarcomatoid cholangiocarcinoma. BMJ case reports. 2018; 2018.

13. Koo HR, Park MS, Kim MJ, Lim JS, Yu JS, Jin H, et al. Radiological and clinical features of sarcomatoid hepatocellular carcinoma in 11 cases. Journal of computer assisted tomography. 2008; 32: 745-9.

14. Lambin P, Leijenaar RTH, Deist TM, et al. Radiomics: the bridge between medical imaging and personalized medicine. Nature reviews Clinical oncology. 2017; 14: 749-62.

15. Castellano G, Bonilha L, Li LM, Cendes F. Texture analysis of medical images. Clinical radiology. 2004; 59: 1061-9.

16. Miles KA, Ganeshan B, Hayball MP. CT texture analysis using the filtration-histogram method: what do the measurements mean? Cancer imaging : the official publication of the International Cancer Imaging Society. 2013; 13: 400-6.

17. Lubner MG, Smith AD, Sandrasegaran K, Sahani DV, Pickhardt PJ. CT Texture Analysis: Definitions, Applications, Biologic Correlates, and Challenges. Radiographics : a review publication of the Radiological Society of North America, Inc. 2017; 37: 1483-503.

18. Ganeshan B, Miles KA. Quantifying tumour heterogeneity with CT. Cancer imaging : the official publication of the International Cancer Imaging Society. 2013; 13: 140-9.

19. Fan Y, Chen C, Zhao F, Tian Z, Wang J, Ma X, et al. Radiomics-Based Machine Learning Technology Enables Better Differentiation Between Glioblastoma and Anaplastic Oligodendroglioma. Front Oncol. 2019; 9: 1164.

20. Lv W, Yuan Q, Wang Q, Ma J, Feng Q, Chen W, et al. Radiomics Analysis of PET and CT Components of PET/CT Imaging Integrated with Clinical Parameters: Application to Prognosis for Nasopharyngeal Carcinoma. Molecular imaging and biology : MIB : the official publication of the Academy of Molecular Imaging. 2019; 21: 954-64.

21. Iasonos A, Schrag D, Raj GV, Panageas KS. How to build and interpret a nomogram for cancer prognosis. Journal of clinical oncology : official journal of the American Society of Clinical Oncology. 2008; 26: 1364-70.

22. Balachandran VP, Gonen M, Smith JJ, DeMatteo RP. Nomograms in oncology: more than meets the eye. The Lancet Oncology. 2015; 16: e173-80.

23. Yuan C, Wang Z, Gu D, Tian J, Zhao P, Wei J, et al. Prediction early recurrence of hepatocellular carcinoma eligible for curative ablation using a Radiomics nomogram. Cancer imaging : the official publication of the International Cancer Imaging Society. 2019; 19: 21

24. Ji GW, Zhu FP, Zhang YD, Liu XS, Wu FY, Wang K, et al. A radiomics approach to predict lymph node metastasis and clinical outcome of intrahepatic cholangiocarcinoma. European radiology. 2019; 29: 3725-35.

25. Zhu HB, Zheng ZY, Zhao H, Zhang J, Zhu H, Li YH, et al. Radiomics-based nomogram using $\mathrm{CT}$ imaging for noninvasive preoperative prediction of early recurrence in patients with hepatocellular carcinoma. Diagnostic and interventional radiology (Ankara, Turkey). 2020.

26. Zhou X, Luo Y, Peng YL, Cai W, Lu Q, Lin L, et al. Hepatic perfusion disorder associated with focal liver lesions: contrast-enhanced US patterns--correlation study with contrast-enhanced CT. Radiology. 2011; 260: 274-81.

27. Nioche C, Orlhac F, Boughdad S, Reuze S, Goya-Outi J, Robert C, et al. LIFEx: A Freeware for Radiomic Feature Calculation in Multimodality Imaging to Accelerate Advances in the Characterization of Tumor Heterogeneity. Cancer research 2018; 78: 4786-9.

28. Youden WJ. Index for rating diagnostic tests. Cancer. 1950; 3: 32-5

29. Fitzgerald M, Saville BR, Lewis RJ. Decision curve analysis. Jama. 2015; 313: 409-10.

30. Zhou X, Ning Q, Jin K, Zhang T, Ma X. Development and validation of a preoperative nomogram for predicting survival of patients with locally advanced prostate cancer after radical prostatectomy. BMC cancer. 2020; 20: 97.
31. Sauerbrei $\mathrm{W}$, Royston $\mathrm{P}$, Binder $\mathrm{H}$. Selection of important variables and determination of functional form for continuous predictors in multivariable model building. Statistics in medicine. 2007; 26: 5512-28.

32. Lu J, Xiong XZ, Li FY, Ye H, Lin YX, Zhou RX, et al. Prognostic Significance of Sarcomatous Change in Patients with Hepatocellular Carcinoma After Surgical Resection. Annals of surgical oncology. 2015; p: S1048-56.

33. Watanabe G, Uchinami H, Yoshioka M, Nanjo H, Yamamoto Y. Prognosis analysis of sarcomatous intrahepatic cholangiocarcinoma from a review of the literature. International journal of clinical oncology. 2014; 19: 490-6.

34. Wang T, Kong J, Yang X, Shen S, Zhang M, Wang W. Clinical features of sarcomatoid change in patients with intrahepatic cholangiocarcinoma and prognosis after surgical liver resection: A Propensity Score Matching analysis. Journal of surgical oncology. 2020; 121: 524-37.

35. Tsou $\mathrm{YK}$, Wu RC, Hung CF, Lee CS. Intrahepatic sarcomatoid cholangiocarcinoma: clinical analysis of seven cases during a 15-year period. Chang Gung medical journal. 2008; 31: 599-605.

36. Liao $\mathrm{SH}, \mathrm{Su} \mathrm{TH}$, Jeng YM, Liang PC, Chen DS, Chen $\mathrm{CH}$, et al. Clinical Manifestations and Outcomes of Patients with Sarcomatoid Hepatocellular Carcinoma. Hepatology. 2019; 69: 209-21.

37. Wu L, Tsilimigras DI, Farooq A, Hyer JM, Merath K, Paredes AZ, et al. Management and outcomes among patients with sarcomatoid hepatocellular carcinoma: A population-based analysis. Cancer. 2019; 125: 3767-75.

38. Davnall F, Yip CS, Ljungqvist G, Selmi M, Ng F, Sanghera B, et al. Assessment of tumor heterogeneity: an emerging imaging tool for clinical practice? Insights into imaging. 2012; 3: 573-89.

39. Casadei-Gardini A, Orsi G, Caputo F, Ercolani G. Developments in predictive biomarkers for hepatocellular carcinoma therapy. Expert review of anticancer therapy. 2020; 20: 63-74.

40. Ji GW, Zhu FP, Xu Q, Wang K, Wu MY, Tang WW, et al. Radiomic Features at Contrast-enhanced CT Predict Recurrence in Early Stage Hepatocellular Carcinoma: A Multi-Institutional Study. Radiology. 2020; 294: 568-79.

41. Akai H, Yasaka K, Kunimatsu A, Nojima M, Kokudo T, Kokudo N, et al. Predicting prognosis of resected hepatocellular carcinoma by radiomics analysis with random survival forest. Diagnostic and interventional imaging. 2018; 99: 643-51.

42. Zheng BH, Liu LZ, Zhang ZZ, Shi JY, Dong LQ, Tian LY, et al. Radiomics score: a potential prognostic imaging feature for postoperative survival of solitary HCC patients. BMC cancer. 2018; 18: 1148.

43. Chen S, Zhu Y, Liu Z, Liang C. Texture analysis of baseline multiphasic hepatic computed tomography images for the prognosis of single hepatocellular carcinoma after hepatectomy: A retrospective pilot study. European journal of radiology. 2017; 90: 198-204.

44. Mulé S, Thiefin G, Costentin C, Durot C, Rahmouni A, Luciani A, et al. Advanced Hepatocellular Carcinoma: Pretreatment Contrast-enhanced CT Texture Parameters as Predictive Biomarkers of Survival in Patients Treated with Sorafenib. Radiology. 2018; 288: 445-55.

45. Giunchi F, Vasuri F, Baldin P, Rosini F, Corti B, D'Errico-Grigioni A. Primary liver sarcomatous carcinoma: report of two cases and review of the literature. Pathology, research and practice. 2013; 209: 249-54.

46. Kim DK, Kim BR, Jeong JS, Baek YH. Analysis of intrahepatic sarcomatoid cholangiocarcinoma: Experience from 11 cases within 17 years. World journal of gastroenterology. 2019; 25: 608-21. 